tion d'une sérieuse station expérimentale, tant réclamée, de son vivant, par Meugniot.

Il est vrai que la Commission de la Carpe existe ; elle pourrait, au moins, contrôler les origrines et les procédés d'èlevage. Mais copnbien sommes-nous ayant déclaré nous soumettre à ce contròle, plus efficace que la présentation, en bocal, de quelques sujets remarquables et dénués de garantie? Deux, peut-ètre trois, alors qu'à l'étranger la garantie fonctionne normalement.

J'en reviens à ma question utilitaire : quelle valeur a la théorie des chromosomes, et quel bénéfice pouvons-nous en tirer ?

\title{
JURISPR UDENGE
}

\section{EXPÉUITION DE POISSON VIVANT A UN COMMISSIONNAIRE CHARGÉ DE LA VENTE \\ RESPONSABILITÉ IOES ACCIDENTS POSTÉRIEURS A LA RÉCEPTION}

Par M. Charles GUYOT

Ancien Directeur de l'Ecole Nationale des Eaux et Forèts.

Nous croyons devoir signaler dans ee Bulletin un arrêt de la Cour dappel de Paris ( 7 "Chambrè), du 23 mars 1929 , qui présente un réel intérèt pour les propriétaires d'étangrs, généralement des carpiculteurs, qui expédient leurs produits pour la vente à $u$ intermédiaire sur les lieux de consommation.

Dans l'aftaire soumise à la Cour, des propriétaires avaient envoyé à Paris plusieurs wagons de Poissons à une Société qui se chargeait, moyennant rémunération, de vendre le Poisson vivant et qui devait rendre compte à ses mandants du produit de la vente dans les ふ̌o jours de l'expédition. Des difficuités s'élevèrent pour la règlement des sommes dues. Après un délai beaucoup plus lonğ qu il "élait convenu, la Société, qui avait pris livaison des wagrons expédics sans faire aucune objection ni réserve, prétendit n’avoir icndu fu un liers de Poisson vivant, et ne payer les deux autres tiers quau prix du Poisson mort.

Ces prétentions avaient été admises par le Tribunal civil de la Seine, le 3 novembre 1993, pour le motif que les risques du transport devaient incomber aux expéditeurs el quiacune faute n'élait élablie de la part de la Société commissionnaire.

En appel, les propriétaires firent ressortir que, si les risques du transport étaient à leur charge, la Sociélé à partir du moment de la réception, 
acceptée par elle sans observation ni réserve, était responsable du préjudice qui pouvait résulter de la mauvaise exécution de son mandat. C'était à elle qu'incombait l'obligation de prouver pour quels motifs un déchet excessif s'était produit postérieurement à la réception. C'omme elle n'avait pas fait cette preuve, la Cour admit que la mortalité des deux tiers des Poissons expédiés devait provenir. comme le prétendaient les appelants, de l'organisation défectueuse des viviers dans lesquels la Société entreposait un trop grand nombre de poissons. Elle avait commis, de ce fait, une faute lourde dont elle devait réparation.

En conséquence, conformément aux conclusions des propriétaires expéditeurs, la somme due par la Société devait leur être réclée au cours moyen des Halles de Paris à l'époque de la réception, sauf un abattement de ro \% correspondant à un déchet normal de mortalité.

Au point de vue des principes, c'est très justement que cel arrît dispose que, si l'expédileur a la charge des risques du transport de la marchandise, à dater de la réception, le commissionnaire est responsable des accidents qui peuvent se produire et qui infuent sur les résultats de la vente. Dans ces sortes de ventes, les conventions sont généralement stipulées, entre expéditeurs et vendeurs, suivant les usages du commerce, sans acte écrit, ce qui peut donner lieu à des difficultés, comme dans l'affaire cidessus. Au moins serait-il utile de convenir expressément d'un court délai dans lequel un accusé de réception constatant l'état de la marchandise doit être donné aux propriétaires expéditeurs, à défaut de quoi tous les risques quelconques sont à la charge du commissionnaire.

\section{SOMMAIRE DE L'ARNET}

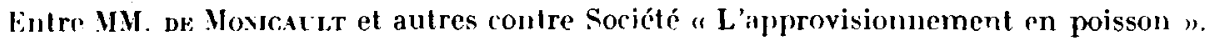

\section{LA Cour,}

... Consillérant que, suivant conventions verbales intervenues er juilled rg22, la société " l'approvisionnement en poisson " s'était chargée, moyennant certaines rémunérations, de vendre le poisson vivant qui lui serait adressé par divers propriétaires d'étangs, dont les appelants, et de rendre compte à ses mandants du produit de ces ventes dans les 3 o jours de l'expédition ;

Qu'il ne paraît pas contesté qu'à la suite de cet accord cincl wagons de poissous vivants furent expédićs à ladite Société, le premier par M. de Monicault, le zo octobre 1922 , le second par M. Gallice, le 25 octobre I923, le Iroisième par $M$. Winckler, le ? novembre 192:, le quatrième ef le cinquième par Y. Pol Roger, le \& novembre i92?;

Que le poids du poisson ainsi expédić a été reconnu a l'arrivée comme étant pour le premier wagon de $\{.0-6 \mathrm{kilos}$, pour le deuxième de 4.9 fio kilos, pour le traisième de 4.935 kilos, pour les quatrième et cinquième de 5.445 kílos ;

Que ce poisson a été reşu vivant par la Société d'approvisionnement qui en a pris livraison, et, pour le wagon de $M$. Gallice en a même accusé réception aussitôt à i'expéditeur avec promesse de règlement rapide, sans formuler aucune objection ni fiuire aucune réserve sur l'śtat de cette marchandise ;

Que cependant, par la suite, les appelants ne purent obtenir le règlement de leurs. 
expéditions, en- tenant compte, comme il en̂t été régulier, de la quantité et de l'espèce du poisson fourni par chacun d'eux;

Que, sur leur mise en demeure, la Société mandataire se contenta alors d'envoyrr au président de l'Union des proprićtaires d'étangs un compte glohal et sommairı, duquel il résultait qu'il avait été vendu seulement $7 . \not 3$ r kil. 5oo de poisson vivanl. cl I4.J7 kil. Ino, c'est-i-dire près des deux liers, de poisson mort;

Considérant que c'est dans ces conditions que les appelants ont assigné la sociélé d'approvisionement en paiement du prix du poisson vivant d'après les cours des Halles. : J'époque où la vente aurait dù être effectuée et sous déduction des frais et commissions légitimement dus ì l'intermédiaire ;

Que le jugement entrepris les a dóboutés de leurs demandes, molif pris de ce qur les risques du transport du poisson incombant aux expediteurs, la sociéts destinatiire n'était que commissionnaire, et de ce qu'aucune faute noitat élablie i l'encontre de Iadite Société ;

Mais considérant que cette décision fait cn l'espèce une inexacte appréciation de: rurconstances de la cause et des droits respectifs des parties :

Que s'il n'est pas douteux, tout d'abord, que les risques du transport du poisson cilaient effectivement a la charge des expéditeurs, il convjent de se souvenir que la róclamation des appelants porte exclusivement sur les quanlités de poissons vivants jrises en charge à l'arrivée par la Société d'approvisjonmement sans aucune objection ni réserve de sa par't ;

Qu'à partir de ce moment, l'obligation de mandalaire salarié de ladile Sociélé lui imposait, non seulement de rendre compte de lat vente d'une marchandise gu'clle asidit rşue vivante, mais la rendait ćgalement responsable du préjudice qui pouvait résulter pour ses mandants de la mauvaje exécution du mandat qu'elle avait assumé ;

Qu'à cet égard, si un déchet normal de mortalité eùt été relativement opposable: aux commeltants sans justification particulière, la proportion des deux tiers relevie dans le compte établissait au contraire, à la charge de li société d'approvisionmement, une présomption de faute lourde dont elle était tenue de se disculper ;

Que le jugement entrepris a cru pouvoir retenir, sur ce point, des rilports amananl de M. Popieul, administrateur-délégué de la Sociélé dapprovisionnement, desłuels ii scmblerait résulter que le poisson expédić aurait souffert au cours du transport;

Mais considérant que des rapports produits aux débats, établis sur des fenilles volantes, ne présentent aucune garantio d'authenticité, que ce sont des attestaliuns tue la Socićté s'est fournjes elle-mème, et que, non seulement les prótendues constiatations relevées dans ces rapports n'ont pas été failes contradictoirement ni même signalés ultérieurement aux intéressés, mais qu'il s'y rencontre des rrreurs formelles ciont la preuve a été rapportéc à l'audience ; qu'il est, au contraire, heaucoup plus rationmel et vraisemblable de penser, avec les apmolants, que le dichel excessif yui s'st produit après réception du poisson par la Société d'approvisionnement provient aniquement de l'organisation défectueuse de ses viviers où elle entreposail un trop frand nombre de poissons, dans des bassins insuffisamment pourvus d'oxygène;

Que des lors la Société d'approvisionnement demeure responsable de lil perte surveunce ef doit le paicment du prix du poisson vivant reçu par elle ;

Qu'cn calculant ce prix au cours moyen des Halles d'octobre el novembre iga, shis déduction des frais el commissions ligitimes, avec un abatlemenl de no correspondant ì un dérhet normal de morlalité, on établit les créances respeclives des appelauts de la manière suivante..., avec les intérets de droit à compter du jour de l'assiquation ;

Par ces motifs, - Infirme le jugement entrepris; condamne la sociéte d'approvi. sionnement à payer respeclivement...; condamne la même socićlè à tous les dépens de première instance et d'appel. 